\title{
Defect-Induced Magnetism in II-VI Quantum Dots
}

\author{
Matheus P. Lima, ${ }^{1, *}$ L. Cabral,${ }^{1}$ Emanuela Margapoti, ${ }^{1}$ Suddhasatta Mahapatra, ${ }^{2}$ Juarez L. F. \\ Da Silva, ${ }^{3}$ Fabian Hartmann, ${ }^{4}$ Sven Höfling, ${ }^{4,5}$ Gilmar E. Marques, ${ }^{1}$ and Victor Lopez-Richard ${ }^{1}$ \\ ${ }^{1}$ Departamento de Física, Universidade Federal de São Carlos, 13565-905 São Carlos, SP, Brazil \\ ${ }^{2}$ Department of Physics, Indian Institute of Technology Bombay, Mumbai 400076, India \\ ${ }^{3}$ Instituto de Química de São Carlos, Universidade de São Paulo, 13560-970 São Carlos, São Paulo, Brazil \\ ${ }^{4}$ Technische Physik, Physikalisches Institut and Röntgen Center for Complex Material Systems (RCCM), \\ Universität Würzburg, Am Hubland, D-97074 Würzburg, Germany \\ ${ }^{5}$ SUPA, School of Physics and Astronomy, University of St Andrews, St Andrews, KY16 9SS, United Kingdom
}

\begin{abstract}
The identification of physical parameters that lead to magnetism in apparent non-magnetic semiconductor systems have continuously challenged our community. In particular, for quantum-dot systems, they are key factors that contribute to their magneto-optical properties. We report, experimentally, optical evidences of induced nano-magnetism in non-magnetic CdSe quantum-dots and assess theoretically the role played by charged and uncharged vacancies. The analysis of these effects rests upon both the chemical and strain environments where the quantum dots are embedded. The interplay of spin-orbit interaction with built-in axial strains has been demonstrated to be a key factor for the magnetic moment alignment. This has been achieved in this paper by emulating the electronic structure at atomistic levels, considering various defect configurations and taking into account both the quantum dot composition and the influence of the host lattice.
\end{abstract}

Keywords: nano magnetism, quantum dots, CdSe, vacancies

\section{INTRODUCTION}

Localized magnetic moments induced in otherwise seemingly non-magnetic system have attracted the attention of the scientific community due to the possibility of enabling and controlling magnetic responses. The idea, for instance, of inducing ferromagnetism in a system whose bare building blocks have no magnetic response, demands a thorough analysis about the potential source of this effect. Semiconductor thin films ${ }^{1}$ and graphene ${ }^{2}$ have been on the focus of these endeavors. Yet, for a long time already this issue has pervaded the study of inorganic quantum dots $(\mathrm{QDs})^{3-8}$ wherein the origin of the magnetism is still profusely debated and has even risen to the level of a potential universal behavior, as stated in Ref. 6.

Moreover, the topic of induced magnetism in nonmagnetic material systems is an active research field. In colloidal II-VI QDs, the emergence of paramagnetic centers as source of the magnetism has been controlled by manipulating their surface chemistry ${ }^{5,6,9-11}$ or charge ${ }^{3}$. Also, the prominent exchange interaction in this type of nano-particles ${ }^{7}$ must be considered to be an essential ingredient for the magnetic ordering. Unexpected magnetism has also been found in caped gold nano-particles with surface-bound molecules as reported in Ref. 12, anticipating applications in catalysis and biomedicine. Even in the case of pristine $\mathrm{Au}$ nano-particles, ferromagnetic ordering has been predicted theoretically from first-principles calculations ${ }^{13}$, ascribed to surface effects. This ferromagnetism was later confirmed experimentally by magnetization measurements as described in Ref. 14 . Yet, in all the examples highlighted above, the optical vestiges of this magnetic ordering are missing.

In this paper, we explore the magnetism that arises from defects in self-assembled CdSe QDs grown within a ZnSe host lattice and assess the relevance of strain fields and spin-orbit effects for its persistence. The purpose of our study is to deliver insights to the magnetism of these seemingly non-magnetic nanoscopic systems that can be detected optically in their micro photoluminescence (micro-PL) and traced down to the spindynamics characterized by their polarized light emission resolved in time. The nature of the effect is discussed by using an $a b$ initio approach for the electronic structure simulations that considers both the CdSe QD and the surrounding ZnSe environment, and hence explicitly takes into account effects from the quantum confinement, interface reconstructions, and the stress induced by the lattice parameter mismatch between the core and surrounding regions.

By means of a systematic analysis on the potential source of paramagnetic centers, we demonstrate that monovacancies lead to the emergence of localized magnetic moments, whereas the lattice deformation plays a key role in its energetic stability. More specifically, we demonstrated that: (i) single vacancies lead to localized magnetic moments ranging from 0 to $3 \mu_{B}$ being the neutral Se vacancy the only non-magnetic defect whereas the neutral Cd vacancy is the most likely source of paramagnetic centers, with $m=2 \mu_{B}$; (ii) the compression of the CdSe chemical bonds induced by the lattice parameter mismatch with the ZnSe host is responsible for decreasing in $50 \%$ the formation energy of defects when compared to the pristine unstrained structures; (iii) the selfassembling process generates an axial strain, and our simulations reveal that the Magnetic Anisotropy Energy (MAE) is highly sensitive to it, presenting an increase from 7.0 to $10.4 \mathrm{meV}$ for anisotropic axial strains around $1.25 \%$. The buildup of such a strain field will favor the alignment of the localized magnetic moments. 


\section{METHODS AND RESULTS}

The QDs investigated in this work are composed of CdSe layers embedded in a ZnSe matrix, and were synthesized by molecular-beam-epitaxy (MBE) ${ }^{15}$. Two monolayers of CdSe, were grown on top of a $50 \mathrm{~nm}$ thick ZnSe barrier on top of a $300 \mu \mathrm{m}$ thick GaAs substrate. The QDs are protected by the deposition of a $50 \mathrm{~nm}$ thick ZnSe cap layer. The QDs formed have a height of 1.5$2 \mathrm{~nm}$ and $8-20 \mathrm{~nm}$ in-plane extension. The large QDs densities and the size distribution lead to a broad photoluminescence. In order to resolve a single QD, the number of excited dots were reduced by fabrication of mesas structures (down to $50 \mathrm{~nm}$ in size). Accordingly with the size of the mesa fabricated, the number of active QDs carried by the mesa will change. The single QD was measured only from the $100 \mathrm{~nm}$ and the $50 \mathrm{~nm}$ mesas. For the micro-PL measurements that detect the single QD emission, the sample was excited by a GaN solid state continuous-wave laser emitting at $405 \mathrm{~nm}$. The magnetic properties of QDs have been also studied by using another set-up, where an external magnetic field was applied. In this set of measurements an argon ion $\left(\mathrm{Ar}^{+}\right)$ laser, emitting in the visible range, was used as excitation source. In most of the measurements the $458 \mathrm{~nm}$ line was selected. The measurements were collected when the sample was cooled to $\sim 2 \mathrm{~K}$. This temperature was obtained by flowing liquid He into the sample chamber through a pin-hole. In order to study more systematically the degree of circular polarization of the QDs a $50 \mathrm{kHz}$ photoelastic modulator operating as a $\lambda / 4$-plate in combination with a linear polarizer was placed in front of the monochromator. For the time resolved measurements, an $\mathrm{Ar}^{+}$ion laser beam has been used to pump the Ti-Saphire laser operating in the picosecond mode. The infrared light, emitted from the Ti-Sa laser, is doubled by a non-linear BBO crystal. Thus a visible laser light is generated. Finally, by the use of a streak camera the lifetime of QDs, excited by this light, were analyzed.

The cross sections of transmission electron microscopy images of the QDs are displayed in Figure 1 (a). The $\mathrm{ZnSe}$ spacer between the two QD layers is $3 \mathrm{~nm}$ allowing an effective vertical electronic coupling and an axial strain relaxation along the growth direction. The map of the lattice constant, along the growth direction, has been estimated from high-resolution TEM imaging as shown in Fig. 1 (b).

The optical response was assessed by exciting the samples with an argon ion laser and the micro-PL was measured in a cryostat with the temperature ranging $2-5 \mathrm{~K}$. The circular polarized QD emission was studied varying the magnetic field strength. In Figures 1 (c)-(d), the emission spectra from single QDs (labeled QD1, QD2, and QD3) have been displayed for various fields. The broad and asymmetric peak is attributed to spectral diffusion as produced by local electric fields due to trapped charges in the vicinity of the quantum $\operatorname{dots}^{16-18}$.

Note that a peak splitting can be clearly observed at
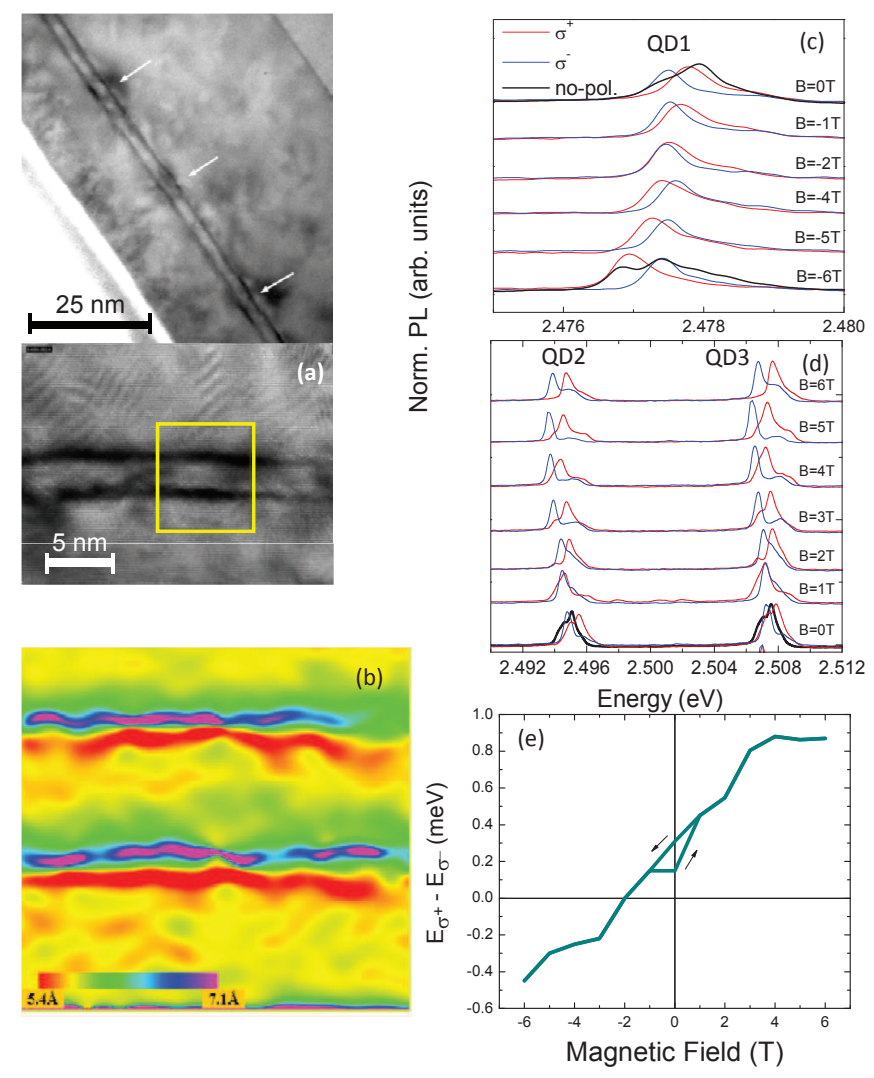

FIG. 1. (color online.) (a) TEM images of two monolayers of CdSe quantum dots taken from Ref. 19. (b) Color-map of the lattice constant in the growth direction estimated from high-resolution TEM imaging. (c) and (d) micro-PL spectra of three different quantum dots at various fields for circular polarized detection. Unpolarized emission spectra have been also added for certain fields. (e) Corresponding Zeeman splitting as a function of the magnetic field.

$B=0$ even for the unpolarized detection. This can unambiguously be linked to the Zeeman splitting as proven when overlapping the result with the circular polarized measurements. The energy splitting for the emission lines from QD2 is shown in panel (e). Note that at $B=0$ a finite splitting can be observed along with a hysteresis that indicates a potential magnetic ordering. It is important to stress that, unlike the two QD layer system, where the magnetism was unveiled, a single layer of QDs, grown under the same conditions, showed no intrinsic magnetic response. So, no traces of this effect were found in the micro-PL of the single QD layer as presented and discussed in Ref. 19.

An additional complementary confirmation of the presence of an intrinsic magnetism can be obtained by assessing the dynamics at the spin-split ground states. The transients of time-resolved PL with linear excitation, measured at $B=0$ are depicted in Figure 2 for two circularly polarized detection. For a spin-split regime, produced by an intrinsic magnetic field, the spin dynamics leading to Rabi-like flopping can be emulated by a 


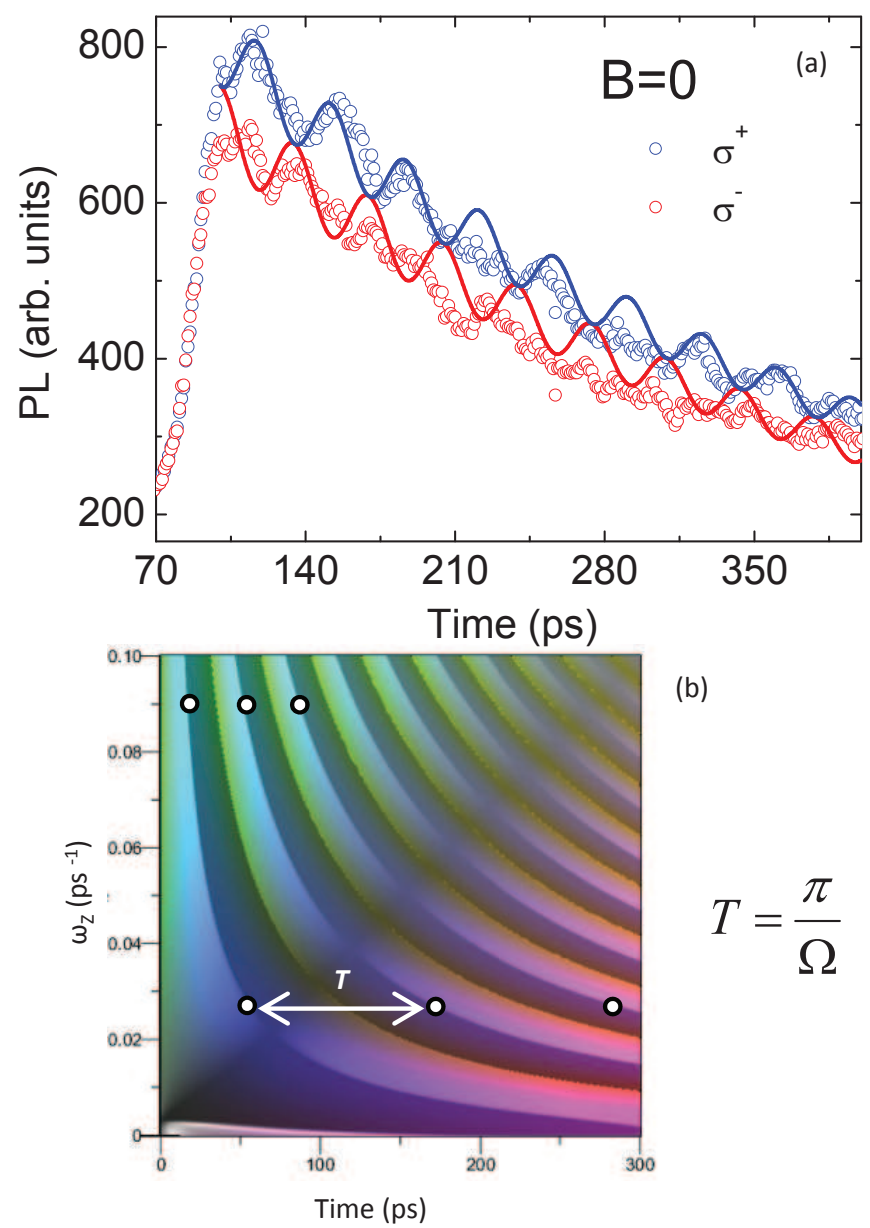

FIG. 2. (color online.) (a) Measured time resolved integrated intensity at $B=0$ and linear polarized excitation, for $\sigma^{+}$ and $\sigma^{-}$circular polarized emissions, denoted by blue and red open circles, respectively. Solid curves indicate the emulated evolution of the spin density for each spin-polarization. (c) Emulated degree of circular polarization as function of time and $\omega_{Z}$.

simplified model

$$
i \hbar \frac{d}{d t}\left(\begin{array}{c}
s_{+} \\
s_{-}
\end{array}\right)=\left(\begin{array}{cc}
\hbar \omega_{z}-i \gamma & \alpha \\
\alpha & -\hbar \omega_{z}-i \gamma
\end{array}\right)\left(\begin{array}{c}
s_{+} \\
s_{-}
\end{array}\right)
$$

that considers a spin-decoherence mechanism through the parameter $\alpha$, that relates to a weak spin admixture through spin-orbit coupling and a life-time broadening $\gamma$ of the Zeeman splitting term, $\hbar \omega_{z}=1 / 2 g^{*} \mu_{B} B^{i n}$, that leads to the wavefunction decay. This will define solutions expressed by

$$
s_{+(-)}(t)=e^{-\gamma t}\left[\cos (\Omega t)-(+) i \frac{\hbar \omega_{z}-(+) \alpha}{\hbar \Omega} \sin (\Omega t)\right],
$$

with $\Omega=\sqrt{\omega_{z}^{2}+\alpha^{2} / \hbar^{2}}$. By considering the linear polarized excitation, where $s_{+}(0)=s_{-}(t)$, the spin oscillations given by $\left|s_{+(-)}\right|^{2}$ are depicted in Figure 2 (a) corresponding to the parameters: $\omega_{z}=0.09 \mathrm{ps}^{-1}$, $\gamma_{z}=0.99 \mu \mathrm{eV}$, and $\alpha=3.95 \mu \mathrm{eV}$. The periodicity of the spin-oscillation can be assessed as plotted in Figure 2 (b) where the degree of circular polarization $\left(\left|s_{+}\right|^{2}-\left|s_{-}\right|^{2}\right) /\left(\left|s_{+}\right|^{2}+\left|s_{-}\right|^{2}\right) /$ has been displayed. The period, given by $T=\pi / \Omega$ is defined by the spin-splitting generated by the internal field, $B^{i n}$, and is related to the value of the effective $g$-factor, $g^{*}$, which is susceptible to both the confinement profile, asymmetries, and exchange interaction, as described in Ref. 20, leading to an ambiguity in the definition of $B^{i n}$. However, the fitting value of $\omega_{z}=0.09 \mathrm{ps}^{-1}$ used in Figure 2 corresponds to an energy splitting $\Delta E=2 \hbar \omega_{z}=0.12 \mathrm{meV}$, which is of the order of the detected spin splitting in the circularly resolved micro-PL emission at $B=0$ depicted in Figure $1(\mathrm{e})$.

A variety of approaches have been presented in the literature to puzzle out the nature of this intrinsic magnetism that emerges in a variety of seemingly nonmagnetic systems, in particular $\mathrm{QDs}^{21,22}$. They all ascribe the effect to the appearance of vacancies and the local magnetic moments attributed to them as the source of paramagnetic centers. A drawback of previous approaches used to simulate self-assembled QDs, is the omission of the effects of the surrounding host matrix. In the present approach, the effects of a surrounding host ZnSe matrix on a CdSe core region was explicitly taken into account, namely: (i) the quantum confinement, (ii) the interface reconstructions, and (iii) the strain induced by the lattice mismatch. These ingredients were demonstrated to be determining factors for the generation and energetic stability of magnetized defects.

In our ab initio investigation, the calculations were performed based on the Density Functional Theory $(\mathrm{DFT})^{23,24}$. We employed the spin-polarized Generalized Gradient Approximation (GGA), making use of the semilocal exchange correlation functional proposed by Perdew-Burke-Erzenhof $(\mathrm{PBE})^{25}$. The Kohn-Sham equations were solved using the Projected Augmented Wave (PAW) method ${ }^{26,27}$ as implemented in the Vienna $a b$ initio Simulation Package (VASP - version 5.4.1 $)^{28,29}$. The employed valence configurations are $5 s^{2} 4 d^{10}, 4 s^{2} 3 d^{10} 4 p^{4}$ and $3 d^{10} 4 s^{2}$ for Cd, Se and Zn, respectively. Also, an energy cutoff of $369 \mathrm{eV}$, a force criterion of $0.0250 \mathrm{eV}^{-1}$, and a Gaussian smearing of $0.001 \mathrm{eV}$ were used. Only the $\Gamma$ point was considered for sampling the Brillouin zone. For charged systems, due to the long range character of the Coulomb interactions ${ }^{30}$, we used the monopole corrections as formulated by Neugebauer et $a l^{31}$ to avoid the spurious selfimage interactions introduced by the periodic boundary conditions. The Magnetic Anisotropy Energy (MAE) ${ }^{32}$ was calculated by $M A E=E_{\|}-E_{\perp}{ }^{33}$, where $E_{\|}\left(E_{\perp}\right)$ is the configuration with the spins parallel (perpendicular) to the easy magnetization $(z)$ axis. For the evaluation of the MAE, the spin-orbit coupling (SOC) was taken into account through the relativistic scalar effects within the non-collinear approach ${ }^{34,35}$. 
The QDs were simulated with a periodic cubic supercell comprised by 216 atoms (38 Cd, $108 \mathrm{Se}$, and 70 $\mathrm{Zn})$. The side of the supercell $(L)$ was defined from the lattice parameter of the host material (ZnSe), being $L=3 \times a_{0}^{\mathrm{ZnSe}}$, wherein the equilibrium lattice parameter $a_{0}^{\mathrm{ZnSe}}=5.74 \AA$ was obtained from the $a b$ initio calculations. The investigated point defects are monovacancies of $\mathrm{Cd}$, Se and $\mathrm{Zn}$. The thermodynamic stability was analyzed from the Formation Energy $\left(E_{F}\right)^{36-38}$, given by

$E_{F}(\alpha, q)=E_{d}(\alpha)-E_{p}(b u l k)-\sum_{i=1} n_{i} \mu_{i}+q\left(\mu_{E}+E_{V B M}\right)$,

(3)

where $E_{d}(\alpha)$ and $E_{p}(b u l k)$ are the total energies of the supercell with a defect indexed by $\alpha$, and the pristine material, respectively. Each specie has been indexed through $i=\mathrm{Cd}$, Se and $\mathrm{Zn}$, and $n_{i}$ represents the number of inserted $\left(n_{i}>0\right)$ or removed $\left(n_{i}<0\right)$ atoms, while $\mu_{i}$ is the chemical potential. The electronic chemical potential, $\mu_{E}$, is the energy distance measured from the top of the valence band, $\left(E_{V B M}\right)$, of the pristine material, and $q=0, \pm 1$ represents the charge excess.

In order to investigate the possibility of defects inducing magnetic moments, characterized by the spin density $\Delta \rho=\rho_{\uparrow}(\mathbf{r})-\rho_{\downarrow}(\mathbf{r})$, we simulated vacancies of $\mathrm{Cd}, \mathrm{Zn}$ and Se in various positions searching for spatially localized paramagnetic centers. In Figure 3, the most relevant neutral defects $(q=0)$ are depicted, as well as their respective isosurfaces for $\Delta \rho$. From a comparative analysis, considering all investigated defects, we found that the configurations presented in Figure 3 form a good sampling capturing the main characteristics of vacancies in the QD. According to Figure 3, Cd vacancies are considered into two different positions: (i) close to the QD center, labeled as Cd-in and (ii) close to the QD edge, denoted as Cd-edge. [see the panels (a) and (b)]. Also, a Zn vacancy in the host material (Zn-matrix) was considered, as depicted in Figure 3 (c). Despite the possibility of creating Se vacancies inside, at the edge, or outside the QD, they do not generate localized magnetic moments, thus we will only discuss the configuration with the lowest energy, namely Se-in, as shown in Figure 3 (d). From these simulations, an intriguing $d^{0}$ magnetism was predicted induced by monovacancies created at the cationic site, e.g. removing $\mathrm{Cd}$ or $\mathrm{Zn}$. The resulting magnetic moment is mainly localized at the four Se atoms neighboring the defect, with a contribution of the p-orbitals, as can be seen in the spin density isosurfaces depicted in Figures 3 (a-c). On the other hand, neutral Se vacancies do not induce localized magnetic moments.

A better understanding of the probability of occurrence of each defect is achieved by contrasting the formation energies among all defects. Furthermore, the occurrence of charged defects is commonly observed for other systems $^{37}$ and we also considered this possibility. Figure 4 provides a clear panorama of the formation energy, local magnetic moments, and charge state for the vacancies discussed in Figure 3, allowing a broad analysis of (a) Cd-in

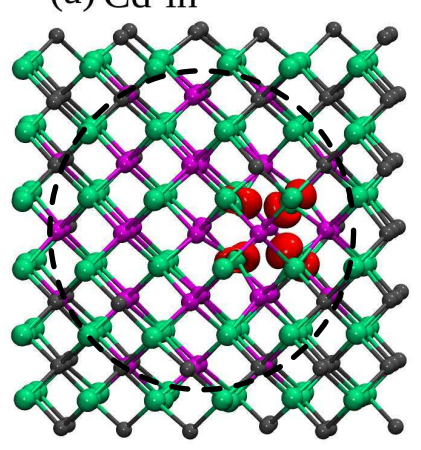

(c) Zn-matrix

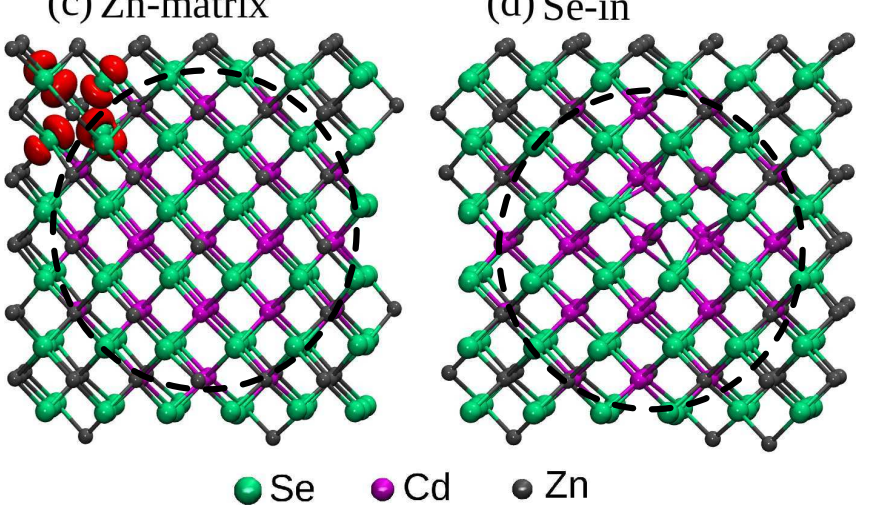

FIG. 3. (color online.) Structures and isosurfaces of the spin density $\left[\Delta \rho=\rho_{\uparrow}(\mathbf{r})-\rho_{\downarrow}(\mathbf{r})\right]$ for neutral CdSe QDs immersed in a ZnSe matrix with vacancies. The isosurfaces for $\Delta \rho=-0.014 \mathrm{e} / \AA^{3}$ are depicted in red. (a) Cd-in: Cd vacancy inside the QD; (b) Cd-edge: Cd vacancy at the edge of the QD; (c) Zn-matrix: Zn vacancy created at the host material; (d) Se-in: Se vacancy inside the QD, which does not present magnetic moment.

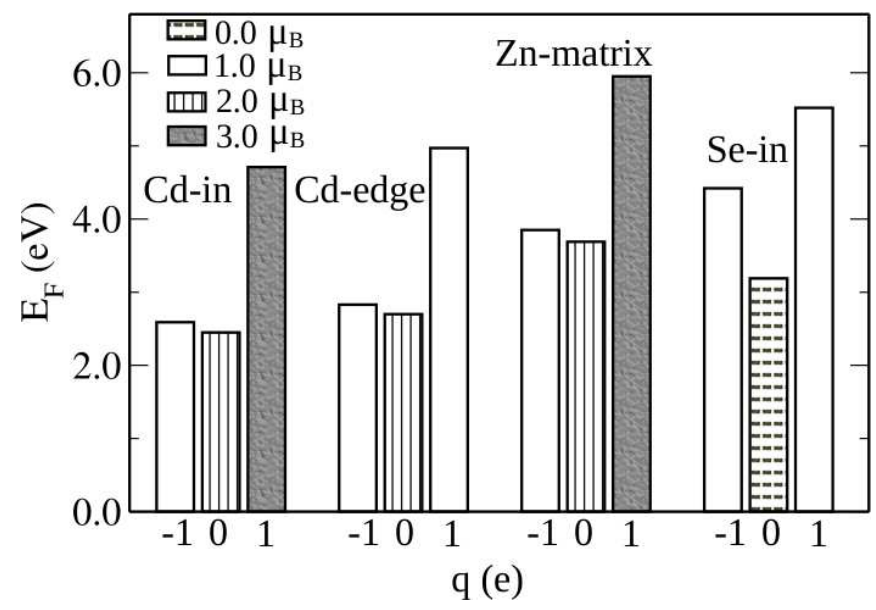

FIG. 4. Calculated $E_{F}$ for the Cd, Se and Zn monovacancies in a CdSe QD immersed in a ZnSe host. Charge states corresponding to $q=-1,0,+1$ were considered (see Eq. (3)). The values for the local magnetic moments were indicated for each configuration.

the investigated point defects. In this figure, the chem- 
ical potentials, $\mu_{i}$, introduced in Eq. (3), were obtained from the free-atoms, and the electronic chemical potential $\mu_{E}$ is measured from the Conduction Band Minimum (CBM). From the data presented in Figure 4, it is notable that the defects induce local magnetic moments ranging from 0 to $3 \mu_{B}$, where the only non-magnetized vacancy is the neutral Se-in structure. Furthermore, the neutral Cd-in vacancy has the lowest formation energy among all defects, which is lower by $250 \mathrm{meV}$ in comparison with the Cd-edge vacancy. The formation of nonmagnetic Se-in vacancy is greater in energy by $740 \mathrm{meV}$ in comparison with the lowest energy configuration, and the Zn-matrix vacancies has the higher formation energy among all, thus highly improbable to occur.

The calculated formation energies of vacancies in CdSe/ZnSe QDs ranges from 2.45 to $5.95 \mathrm{eV}$, with the lower value corresponding to the neutral $\mathrm{Cd}$ vacancy. Aiming to understand how the host material affects the formation energy of monovacancies, we also calculated $E_{F}$ for the Cd vacancy in the unstrained bulk of CdSe with a supercell of 64 atoms, being $4.11 \mathrm{eV}$, which is $67 \%$ higher than $E_{F}$ for Cd-in. Thus, the ab initio simulations demonstrated that the generation of $\mathrm{Cd}$ vacancies is strongly favored inside the CdSe QDs, once its $E_{F}$ decreases if compared to the pristine system.

In order to understand the decrease of formation energy, we should note that a ruling factor determining the thermodynamic stability of defects in the QD is the lattice parameter mismatch between the host, ZnSe, and the core material, CdSe. Such a mismatch generates a compressive strain in CdSe. As a consequence, when a vacancy is created in the core material, an available space

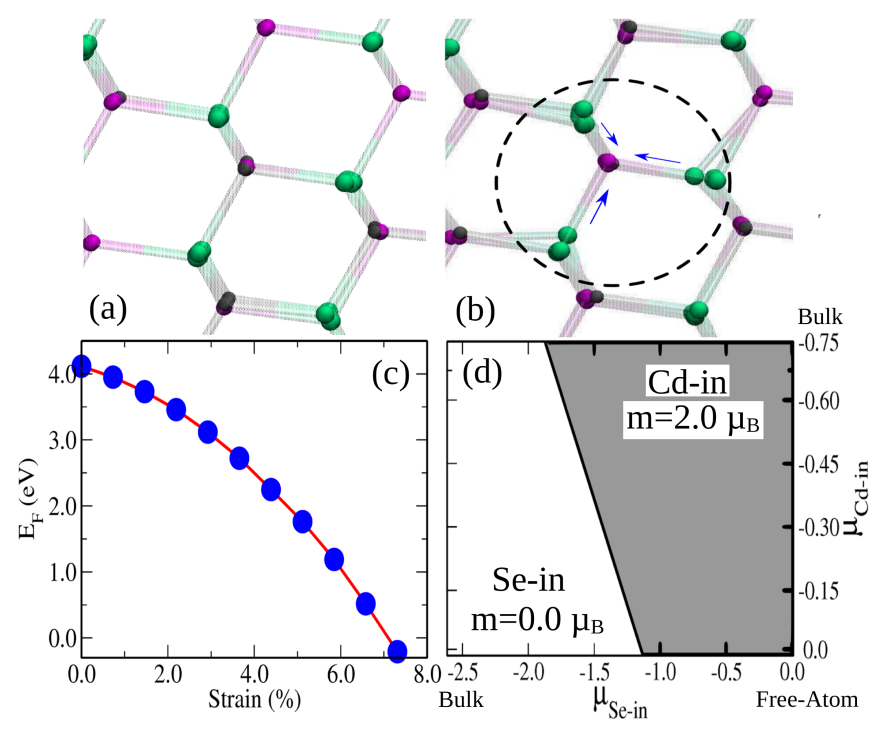

FIG. 5. (color online.) DFT calculations: (a) atoms in a pristine $\mathrm{QD}$ and (b) the same atoms surrounding a $\mathrm{Cd}$ vacancy, where the atomic displacements are depicted by (blue) arrows. Panel (c) presents the evolution of $E_{F}$ with the strain in pristine CdSe. Panel (d) shows a phase diagram, demonstrating the lowest energy defect as a function of $\mu_{C d}$ and $\mu_{S e}$. is opened allowing for a rearrangement of the atomic positions which relieves the strain. This decreases the formation energy in comparison to the vacancies created in unstrained CdSe. Such a behavior is shown in Figure 5. Panel (a) presents the array of atoms of a QD without defects close to the region where a $\mathrm{Cd}$ vacancy will be created, as represented in panel (b). Some distortions in relation to the pristine structure are highlighted in this panel with blue arrows, where the surrounding Se atoms appear dislocated towards the vacancy. In order to completely isolate the effects of a compression in the formation energy of $\mathrm{Cd}$ vacancies, we investigated the way it varies as a function of strain in a pristine CdSe lattice, presented in panel (c) of Figure 5. Such simulations were performed with a supercell of 63 atoms. One may note the pronounced decreasing of the formation energy with strain. The variation in $E_{F}$ reaches $4.0 \mathrm{eV}$ for strain values close to $7.0 \%$. Thus, according to this analysis, if considering QDs of CdSe within a ZnSe lattice, the strain field induced by the lattice parameter mismatch facilitates the formation of defects in the core region.

Note that the Cd-in has a lower $E_{F}$ if compared to the Cd-edge. The energetic preference for defect localized close to the center of the QD is somewhat contrary to the behavior observed in a vast variety of systems. Commonly, there is an energetic preference for defects localized close to the edge ${ }^{39}$. At this point, the peculiar behavior of $\mathrm{CdSe} / \mathrm{ZnSe}$ QDs is addressed to the strain field discussed above. This is a relevant point, once defects localized close to the center of the QD enhance their influence in many QDs properties, such as the optical and magnetic responses.

Focusing again in the monovacancies created in the QDs, it is worth noting that despite the lowest formation energy of $\mathrm{Cd}$-in for the chemical environment (defined by $\mu_{i}$ and $\mu_{E}$ ) analyzed in Figure 4, another low-energy defect is the Se-in. Our total energy simulations predict the possibility to tune the formation energy of these defects by changing the values of $\mu_{C d}$ and $\mu_{S e}$, which represent modifications in the chemical environment in which defects are generated. Notably, it is possible to obtain situations in which the Se-in has the lowest $E_{F}$ through the tuning of $\mu_{i}$. A phase diagram demonstrating the most energetically probable defect as a function of $\mu_{C d}$ and $\mu_{S e}$ is depicted in Figure 5 (d). It is important to stress that the Cd-in defect has a local magnetic moment of $2 \mu_{B}$, whereas the Se-in defect is demagnetized. Thus, the chemical environment in which the vacancies are generated can lead to magnetized or demagnetized defects, which will highly affect the magnetic response of the QDs.

Another environment variable ruling the formation energy of charged defects is the electronic chemical potential $\mu_{E}$. To perform a further analysis, we varied $\mu_{E}$ from the VBM to the $\mathrm{CBM}^{40}$, as shown in Figure 6 . Such simulations demonstrate that neutral defects are the most stable ones. For $\mu_{E}$ close to the VBM, the positively charged defects are at least, $1.18 \mathrm{eV}$ higher in 


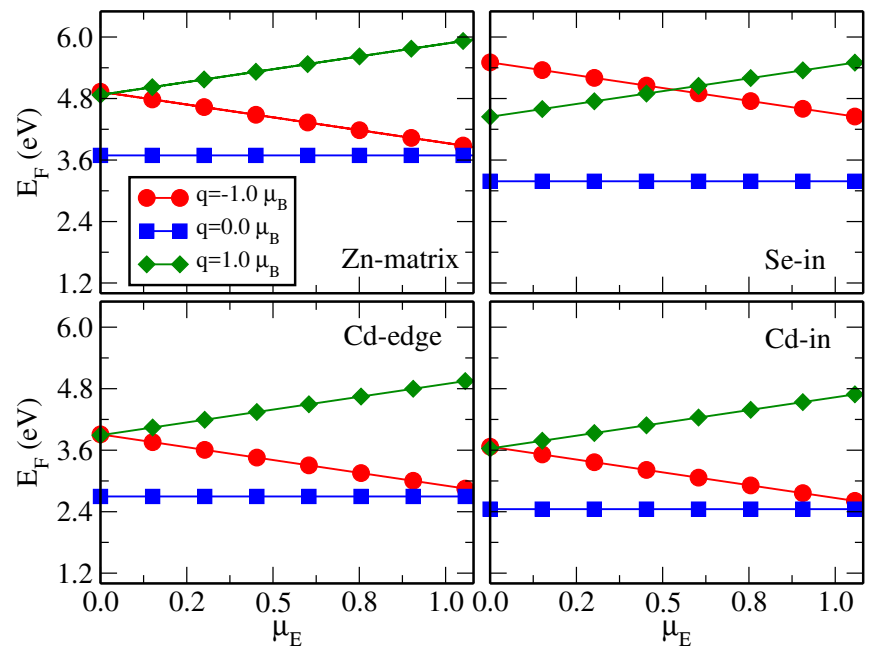

FIG. 6. (color online.) $E_{F}$ as a function of the electronic chemical potential $\mu_{E}$ for $q=-1,0,+1$ [see Eq. (3)].

energy than the neutral ones. For $\mu_{E}$ close to the CBM, the negatively charge defects have $E_{F}$ at least $0.13 \mathrm{eV}$ higher than the neutral ones.

Our $a b$ initio simulations reveal that vacancies are the probable sources of paramagnetic centers, whereas strain fields induced by the lattice parameter mismatch between the core and matrix regions facilitates the formation of defects inside the core region. However, to describe the detected ferromagnetic behavior, the orientation of the local magnetic moments is paramount. In order to investigate this property, we firstly considered the axial asymmetry imposed by the QD selfassembling growth. The preferential spin direction was investigated through the MAE ${ }^{32,33}$ that includes the spin-orbit effects. The data presented in Figure 7 shows the MAE dependence on both axial and hydrostatic strains. The axial strain produces greater contrasts in the MAE, while the hydrostatic strain is almost unperceived. For instance, an axial strain of $1.25 \%$ leads to an increase of one order of magnitude in the MAE, reaching values comparable to those measured in some transition metal compounds ${ }^{41}$. On the other hand, under an axial compressive strain of $-1.00 \%$ the MAE vanishes due to the disappearance of the local magnetic moment (see the inset of Figure 7). Such a high MAE modulation induced by the axial strain indicates a potential source for magnetic ordering induced by the strain anisotropy.

It is well known that the strain in self-assembled quantum dots formed by the Stranski-Krastanov mechanism is non-uniformly. While the wetting layer is pseudomorphically strained, strain relaxation is largest at the top of the islands. A direct evidence of this is seen in the color-map of the c-parameter (lattice constant in the growth direction), estimated from the high-resolution TEM imaging reported in Fig. 1 (b). Considering the fact that the bulk lattice constant of CdSe is $6.05 \AA$, it is clearly observed that within the core of the QDs, the c-parameter is sig-

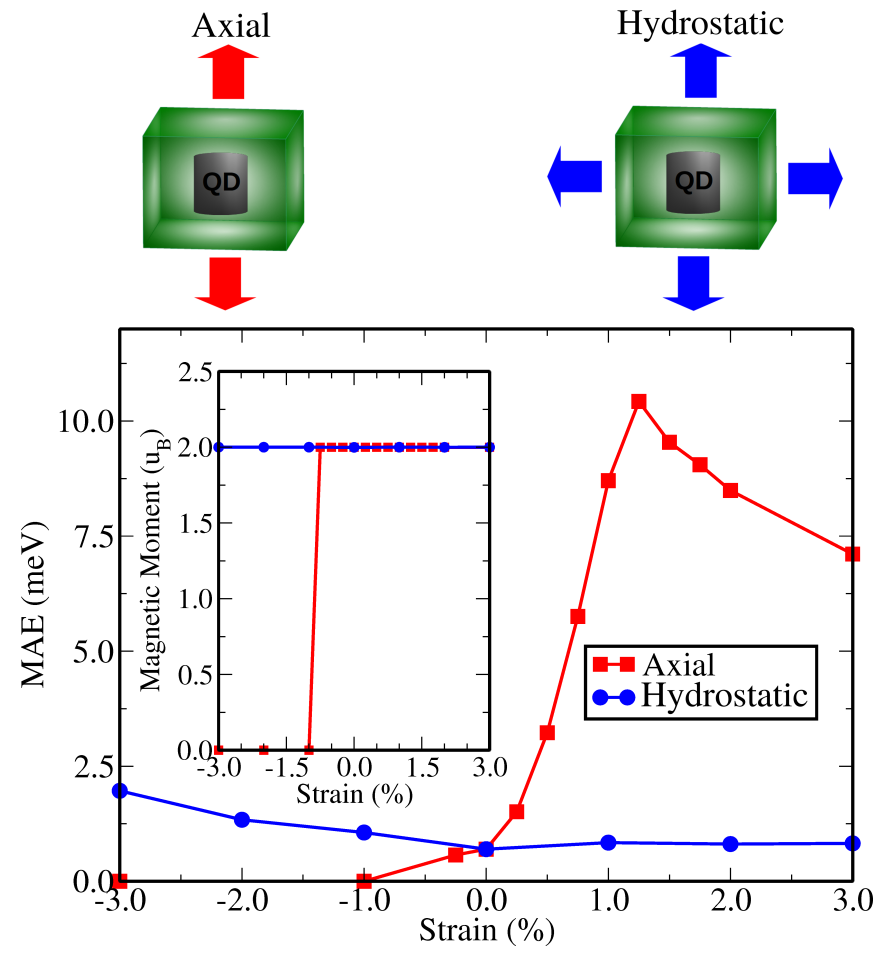

FIG. 7. (color online.) Magnetic Anisotropy Energy and magnetic moment (inset) as function of strain. Compressive and tensile strain in the hydrostatic direction does not change the MAE and the magnetic moment significantly, whereas the major variation was obtained by using the tensile strain for the axial quantization.

nificantly higher (up to $7.1 \AA$ ), suggesting that the CdSe material at the core is highly compressively strained. Moving towards the matrix, the c-parameter reduces, which may be attributed to strain relaxation and/or Zn intermixing of the CdSe QDs.

Also worth noting that the spacer-ZnSe layer close to the CdSe wetting layer is tensile-strained. This is observed by noting that the ZnSe bulk lattice constant is $5.66 \AA$, while the measured c-parameter falls to values down to $5.4 \AA$. Another indirect proof of the anisotropic strain environment of the QDs is provided by the positional-correlation of the QDs in the bilayer (marked by the arrows). The correlation of the QDpositions is driven by anisotropic strain fields ${ }^{42}$.

The induced magnetism breaks the spin degeneracy at zero field leading to a fine-structure splitting due the exchange interaction between the electrons and holes and the magnetic defect. As stated previously, the interplay of spin-orbit interaction and the anisotropic strain buildup is the ground for the magnetic moment polarization. Our ab initio simulations reveal that the emergence of paramagnetic centers in CdSe/ZnSe quantum dots is a consequence of both the exchange and the structural anisotropy splittings of the topmost occupied level of the vacancy levels introduced inside the bandgap by defects. In Figure 8 we show a schematic representation of the 
splittings happening in neutral Cd monovacancy states. Section (i) represents the defect levels occurring in CdSe bulk systems without structural anisotropy that do not present exchange splitting. These threefold degenerate states on the angular momentum are mainly composed of $\mathrm{p}$ orbitals centered in the four Se sites surrounding the monovacancy. On the other hand, when we simulate a Cd monovacancy inside a QD, an exchange splitting was observed of $0.04 \mathrm{eV}$, displayed in Section ii, associated with a structural anisotropy splitting of 0.03 and $0.01 \mathrm{eV}$ as represented in Section iii. Once 4 electrons are occupying these states, the final configuration results in a local magnetic moment of $2 \mu_{B}$. This contribution of defects has clearly been overlooked when discussing the effect of anisotropic electron-hole exchange interaction in the excitonic recombination, as discussed in Refs. 43 and 44

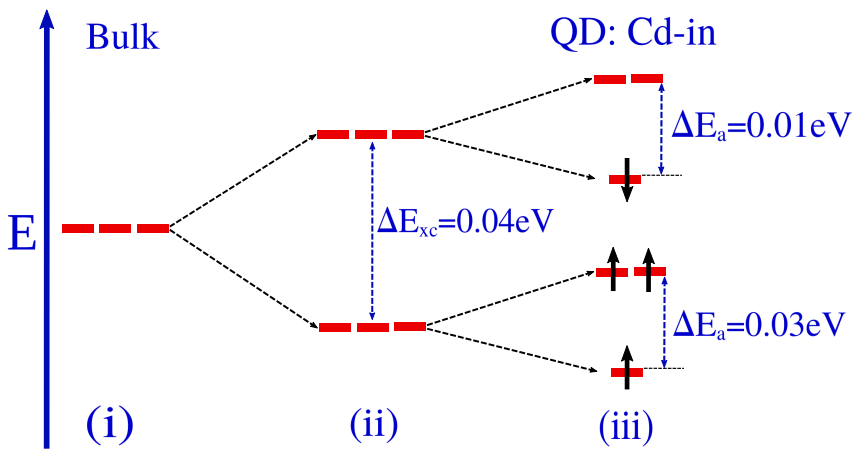

FIG. 8. (color online.) Defect levels introduced by a neutral Cd monovacancy in CdSe systems. (i) defect levels in CdSe bulk. (ii) exchange splitting, and (iii) structural anisotropy splittings happening in CdSe/ZnSe QDs.

\section{CONCLUSIONS}

In summary, in this work we detected and characterized the optical effects of intrinsic magnetism in a seemingly nonmagnetic system. The origin of the localized magnetic moments has been emulated in charged and uncharged non-magnetic CdSe QDs embedded in ZnSe host material. Our formation energy analysis indicates that $\mathrm{Cd}$ vacancies inside the $\mathrm{QD}$ are the preferential defect configuration among all the studied structures. Also, the appearance and vanishing of the magnetic moment is more probable for uncharged systems. The calculated Magnetic Anisotropy Energy indicates that the interplay of spin-orbit interaction with built-in axial strains is a key factor for the magnetic moment alignment.

\section{ACKNOWLEDGEMNTS}

This research was developed using the computational resources of the Centro de Ciências Matemáticas Aplicadas à Indústria (CeMEAI) founded by the Brazilian agency FAPESP. The authors acknowledge the financial support of FAPESP (grants: 2014/19142 - 2, 2014/02112 - 3), CNPq and CAPES. We thank Prof. Eduardo Menéndez-Proupin, from Universidad de Chile, and Prof. Sergio Ulloa, from Ohio University, for their useful insights and fruitful discussions. *mplima@df.ufscar.br

1 H. Ren, G. Xiang, G. Gu, X. Zhang, W. Wang, P. Zhang, B. Wang, and X. Cao, J. Nanomat. 2012, 6 (2012).

${ }^{2}$ Nair R.R., Tsai I.-L., Sepioni M., Lehtinen O., Keinonen J., Krasheninnikov A.V., Castro Neto A.H., Katsnelson M.I., Geim A.K., and Grigorieva I.V., Nat. Commun. 4, 2010 (2013).

${ }^{3}$ S. Neeleshwar, C. L. Chen, C. B. Tsai, Y. Y. Chen, C. C. Chen, S. G. Shyu, and M. S. Seehra, Phys. Rev. B 71, 201307 (2005).

4 S. B. Singh, M. V. Limaye, S. K. Date, and S. K. Kulkarni, Chem. Phys. Lett. 464, 208 (2008).

${ }^{5}$ R. W. Meulenberg, J. R. I. Lee, S. K. McCall, K. M. Hanif, D. Haskel, J. C. Lang, L. J. Terminello, and T. van Buuren, J. Am. Chem. Soc. 131, 6888 (2009).

6 A. Sundaresan and C. Rao, Nano Today 4, 96 (2009).

7 A. Saha and R. Viswanatha, ACS Nano 11, 3347 (2017).

8 V. Proshchenko, A. Karanovich, and Y. Dahnovsky, J. Phys. Chem. C 120, 11253 (2016).

9 M. S. Seehra, P. Dutta, S. Neeleshwar, Y.-Y. Chen, C. L. Chen, S. W. Chou, C. C. Chen, C.-L. Dong, and C.-L. Chang, Adv. Mater. 20, 1656 (2008).
${ }^{10}$ L. Sun, W. Zhou, Y. Liu, Y. Lu, Y. Liang, and P. Wu, Comput. Mater. Sci. 126, 52 (2017).

11 D. Cao, H. Shu, T. Wu, Z. Jiang, Z. Jiao, M. Cai, and W. Hu, Appl. Surf. Sci. 361, 199 (2016).

12 S. Trudel, Gold Bull 44, 3 (2011).

13 W. Luo, S. J. Pennycook, and S. T. Pantelides, Nano Lett. 7, 3134 (2007).

14 V. Tuboltsev, A. Savin, A. Pirojenko, and J. Räisänen, ACS Nano 7, 6691 (2013).

15 S. Mahapatra, T. Kiessling, E. Margapoti, G. V. Astakhov, J. Renner, U. Bass, C. Bougerol, T. Schmidt, A. Bendounan, F. Schmitt, C. Schumacher, L. Worschech, W. Ossau, J. Geurts, L. W. Molenkamp, F. Reinert, A. Forchel, and K. Brunner, Phys. Status Solidi C 4, 3129 (2007).

16 M. J. Ferne, T. Plakhotnik, Y. Louyer, B. N. Littleton, C. Potzner, P. Tamarat, P. Mulvaney, and B. Lounis, J. Phys. Chem. Lett. 3, 1716 (2012).

17 H. D. Robinson and B. B. Goldberg, Phys. Rev. B 61, R5086 (2000).

18 J. Seufert, R. Weigand, G. Bacher, T. Kmmell, A. Forchel, K. Leonardi, and D. Hommel, Appl. Phys. Lett. 76, 1872 (2000). 
19 E. Margapoti, F. M. Alves, S. Mahapatra, T. Schmidt, V. Lopez-Richard, C. Destefani, E. Menéndez-Proupin, F. Qu, C. Bougerol, K. Brunner, A. Forchel, G. E. Marques, and L. Worschech, Phys. Rev. B 82, 205318 (2010).

${ }^{20}$ L. Cabral, F. P. Sabino, V. Lopes-Oliveira, J. L. F. Da Silva, M. P. Lima, G. E. Marques, and V. LopezRichard, Phys. Rev. B 95, 205409 (2017).

21 J.-P. Tang, L. ling Wang, H.-J. Luo, and W.-Z. Xiao, Phys. Lett. A 377, 572 (2013).

${ }^{22}$ W.-Z. Xiao, L. ling Wang, Q.-Y. Rong, G. Xiao, and B. Meng, J. Appl. Phys. 115, 213905 (2014).

23 P. Hohenberg and W. Kohn, Phys. Rev. 136, B864 (1964).

24 W. Kohn and L. J. Sham, Phys. Rev. 140, A1133 (1965).

25 J. P. Perdew, K. Burke, and M. Ernzerhof, Phys. Rev. Lett. 77, 3865 (1996).

26 P. E. Blöchl, Phys. Rev. B 50, 17953 (1994).

27 G. Kresse and D. Joubert, Phys. Rev. B 59, 1758 (1999).

28 G. Kresse and J. Hafner, Phys. Rev. B 48, 13115 (1993).

${ }^{29}$ G. Kresse and J. Furthmüller, Phys. Rev. B 54, 11169 (1996).

30 G. Makov and M. C. Payne, Phys. Rev. B 51, 4014 (1995).

31 J. Neugebauer and M. Scheffler, Phys. Rev. B 46, 16067 (1992).

32 H. Yang, A. D. Vu, A. Hallal, N. Rougemaille, J. Coraux, G. Chen, A. K. Schmid, and M. Chshiev, Nano Lett. 16,
145 (2016).

33 J. Enkovaara, A. Ayuela, L. Nordström, and R. M. Nieminen, Phys. Rev. B 65, 134422 (2002).

34 G. Thiering and A. Gali, Phys. Rev. B 96, 081115 (2017).

35 S. Steiner, S. Khmelevskyi, M. Marsmann, and G. Kresse, Phys. Rev. B 93, 224425 (2016).

36 S.-H. Wei and S. B. Zhang, Phys. Rev. B 66, 155211 (2002).

37 S.-H. Wei, S. B. Zhang, and A. Zunger, J. Appl. Phys. 87, 1304 (2000).

38 S. Chen, J.-H. Yang, X. G. Gong, A. Walsh, and S.-H. Wei, Phys. Rev. B 81, 245204 (2010).

39 G. M. Dalpian and J. R. Chelikowsky, Phys. Rev. Lett. 96, 226802 (2006).

40 S. B. Zhang and J. E. Northrup, Phys. Rev. Lett. 67, 2339 (1991).

41 P. Błoński and J. Hafner, Phys. Rev. B 79, 224418 (2009).

42 G. Springholz, V. Holy, M. Pinczolits, and G. Bauer, Science 282, 734 (1998).

43 H. Htoon, S. A. Crooker, M. Furis, S. Jeong, A. L. Efros, and V. I. Klimov, Phys. Rev. Lett. 102, 017402 (2009).

44 S. V. Goupalov, Phys. Rev. B 74, 113305 (2006). 